\title{
Using "Machine Learning" to make sense of very big and very small data sets
}

\section{J. HELBERT', M. D'AMORE'}

'DLR, Rutherfordstrasse 2, 12489 Berlin, Germany

Recent years have seen two trends that both require new approaches to analyze data. On one hand we have a dramatic increase in the volume of (spectral) data sets from planetary surfaces. Current and future missions are producing large (spectral) data sets that will undoubtedly require many hours, weeks, or months of human scrutiny to organize, extract and interpret the information contained therein.

On the other hand, we have data sets obtained under highly challenging conditions that are sparse and require dedicated efforts to separate noise from actual signal.

Compositional interpretative efforts for both kind of problems can be greatly facilitated by automated classification schemes that are robust and scientifically meaningful. Such schemes would also be an essential element of intelligent remote sensing systems that will operate on, or near, distant planets, moons or asteroids. 